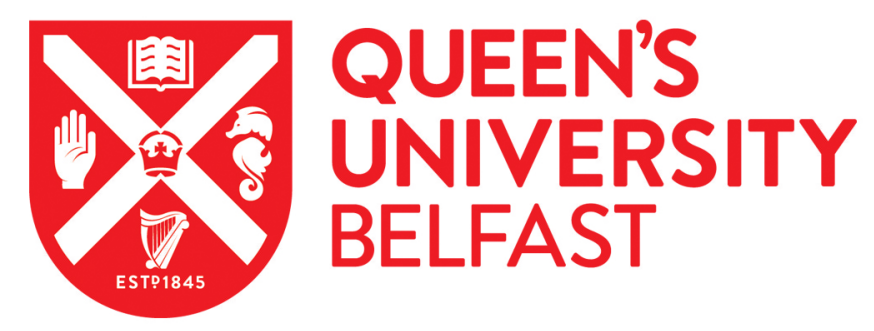

\title{
Psychological factors associated with self-management among adolescents with Type 1 diabetes: a systematic review
}

Martinez, K., Frazer, S. F., Dempster, M., Hamill, A., Fleming, H., \& McCorry, N. K. (2016). Psychological factors associated with self-management among adolescents with Type 1 diabetes: a systematic review. Journal of Health Psychology. https://doi.org/10.1177/1359105316669580

Published in:

Journal of Health Psychology

Document Version:

Peer reviewed version

Queen's University Belfast - Research Portal:

Link to publication record in Queen's University Belfast Research Portal

Publisher rights

Copyright 2016 The Authors

\section{General rights}

Copyright for the publications made accessible via the Queen's University Belfast Research Portal is retained by the author(s) and / or other copyright owners and it is a condition of accessing these publications that users recognise and abide by the legal requirements associated with these rights.

Take down policy

The Research Portal is Queen's institutional repository that provides access to Queen's research output. Every effort has been made to ensure that content in the Research Portal does not infringe any person's rights, or applicable UK laws. If you discover content in the Research Portal that you believe breaches copyright or violates any law, please contact openaccess@qub.ac.uk. 
Running head: Psychological factors and adolescent diabetes

Psychological factors associated with self-management among adolescents with Type 1 diabetes: a review

Kelly Martinez, Sharon F. Frazer, Martin Dempster, Andrea Hamill, Hanora Fleming and Noleen K. McCorry

Queen’s University Belfast, Northern Ireland

Corresponding author:

Martin Dempster

School of Psychology, Queen’s University Belfast, University Road, Belfast, Northern Ireland, BT7 1NN

Tel: +442890975547

Email: $\underline{\text { m.dempster@qub.ac.uk }}$ 
Running head: Psychological factors and adolescent diabetes

\begin{abstract}
This review aims to synthesise the literature examining the psychosocial variables related to self-management (insulin adherence, non-adherence and administration, blood sugar monitoring, dietary behaviour, exercise behaviour) in adolescents with type 1 diabetes. A systematic search of three electronic databases was carried out and, after the application of eligibility criteria, 21 articles were assessed for quality prior to data extraction. Numerous psychological factors were found to be associated with self-management; however, correlations were typically small to moderate. The strongest associations were found between social anxiety and diet (among males); greater intrinsic motivation, conscientiousness and diet; and extraversion and exercise.
\end{abstract}

Keywords:

Diabetes; adolescence; adherence; diet; exercise behaviour 
Type 1 diabetes is a common chronic illness affecting young people in the UK, and its incidence is increasing (Patterson et al., 2012). Although the importance of good glycaemic control to prevent vascular complications (such as retinopathy, neuropathy, and nephropathy) is well recognised, currently in the UK fewer than $25 \%$ of children and young adults achieve the target for long-term glycaemic control ( $\mathrm{HbA}_{1} \mathrm{c}<7.5$ without frequent hypoglycaemia) (Health \& Social Care Information Centre, 2013). Given the plethora of short and long term physical health difficulties related to Type 1 diabetes, it is crucial that young people learn to manage their diabetes effectively, as any decrease in HbA1c decreases the risk of complications (Lind et al., 2009).

However, diabetes management in the adolescent age group presents a more complex set of challenges given the range of physiological, social and emotional changes which occur between childhood and adulthood, including puberty, peer pressure, a desire to be "normal”, identity formation and, often, testing of boundaries set by healthcare professionals, parents and caregivers (Hilliard et al., 2013).

Interventions have focused on enabling young people to better manage their condition. Historically, this has taken the form of educational programmes aimed at teaching specific diabetes management skills and fostering independence with these tasks (for example, carbohydrate counting, blood glucose monitoring), or psychosocial interventions aiming to address self-care and emotional difficulties through a variety of problem-solving and emotion-focused techniques (Peyrot \& Rubin, 2007).

In 2000, the UK National Health Service Health Technology Assessment programme published a systematic review of the literature on the effectiveness of psychosocial and educational interventions for adolescents with diabetes (Hampson et al., 2001). The review described small to medium-sized beneficial effects on diabetes management outcomes, but highlighted numerous weaknesses within the literature - more than half the studies (55\%) had 
no theoretical basis underlying the intervention. A subsequent review (Murphy, Rayman \& Skinner, 2006) attempted to update the existing database and to determine whether the problems identified previously had been addressed. Despite some methodological advances, effects of these reviewed interventions on glycaemic control were limited, with no improvement in the intervening decade. The authors concluded that there was still insufficient evidence to recommend adoption of a particular intervention, and that no intervention had been effective in randomised studies for those with poor glycaemic control.

In light of the problems identified regarding theoretical bases for intervention, Ayling et al. (2014) sought to examine the extent to which theory had informed various interventions and what links this might have had to intervention effectiveness. They found a larger effect size for interventions referencing some theory than for those using none, though use of theory did not predict which interventions were successful. The authors suggest that the lack of theory utilisation for intervention design may be down to a paucity of appropriate theory for young people with Type 1 diabetes. As theory development relies on empirical findings regarding relationships, this paucity may be due to a lack of consensus about which psychological variables are related to self-care and $\mathrm{HbA}_{1 \mathrm{c}}$.

A recent systematic review (Neylon et al., 2013) partially addressed this by providing a narrative synthesis of inter and intra-personal factors related to metabolic control in the adolescent and younger adult (up to 39 years old) age group. However, it is unclear from this review which psychosocial factors exclusively within the adolescent relate to good selfmanagement, something which is of importance as adolescents become more independent in their diabetes management commensurate with the aforementioned transitions and developmental tasks which occur within this age group.

The current review seeks to identify the within-adolescent psychological factors that are associated with self-management behaviours, specifically for adolescents with Type 1 
Running head: Psychological factors and adolescent diabetes

diabetes. Within-adolescent factors means any psychosocial factor that 'resides' within the adolescent. For example, this would include an adolescent's self-report (perception) of parental conflict, but exclude parent reports of the same variable. Unlike the review by Neylon et al. (2013), this review will not employ a lower date limit in an effort to include all existing research.

\section{Method}

\section{Study Selection}

Three electronic databases (CINAHL, MEDLINE and PsycINFO) were searched on $29^{\text {th }}$ August 2014 for relevant articles. Searches encompassed terms related to self-care behaviours such as diet, exercise and blood glucose monitoring. Thesaurus terms (or subject headings) were used instead of keywords, to improve the search.

The thesaurus of each database contained subject headings, which were used as search terms; therefore there were slight variations in the specific search terms used between individual databases. In the search terms below '+' indicates subject headings which were expanded to make the search as broad as possible so as not to omit relevant articles.

In CINAHL, the following thesaurus search terms were used:

Diabetes Mellitus, Type 1 AND (Self Care+ OR Diet+ OR Diabetic Diet OR Eating OR Exercise+ OR Health Behaviour+) AND (Behaviour and Behaviour Mechanisms+ OR Psychological Processes and Principles+ OR Disciplines, Tests, Therapy, Services+ OR Health Education+).

In Psychinfo, the thesaurus search terms used were Diabetes Mellitus AND (Self Management+ OR Self Instructional Training+).

In Medline, the thesaurus search terms used were: Diabetes Mellitus, Type 1 AND Self Care+ AND (behaviour and behaviour mechanisms+ OR psychological phenomena and processes+ OR behavioural disciplines and activities+). 
All results were limited to studies written in the English language, with an adolescent or young adult population. A total of 1310 articles resulted from the three database searches.

\section{Eligibility Assessment}

Duplicates of studies from the database searches were removed $(n=174)$, and article abstracts were reviewed according to pre-determined eligibility criteria. To be eligible for inclusion in this review, a study was required to be available in the English language, a primary research article (i.e. not a review article), with a focus on adolescents with Type 1 diabetes, include diabetes self-management behaviours as an outcome measure, and contain quantitative research examining the associations between psychosocial variables and diabetes self-management behaviour. If an abstract did not meet these criteria, the article was excluded at this stage ( $n=686)$. The remaining articles were retrieved in full text format, and the same eligibility criteria applied. Both the abstract and full text reviews were completed by two reviewers working independently, with disagreements resolved by consensus. A total of 21 articles were considered eligible for the review (see Figure 1 for flow chart).

\section{Quality Assessment}

All included studies were quality assessed using relevant sections of the Effective Public Health Practice Project (EPHPP, 2009), a quality assessment tool for quantitative studies. Given the aim of the study, correlational designs were most likely to be included, yet most existing quality assessment tools are designed for intervention studies. Therefore, the EPHPP tool was used as it was identified as the one most easily adaptable for correlational designs. Consequently, quality assessment primarily focused on areas of external validity: selection bias, data collection methods, and withdrawals / drop outs, along with two additional questions on sample size developed by the research team.

\section{Data Extraction and Analysis}


Correlation coefficients were extracted but, due to the heterogeneity of the psychological variables measured, a meta-analysis was not possible. The studies, therefore, are reviewed in a narrative synthesis, loosely divided between two categories - emotional and cognitive variables - to facilitate comparison.

\section{Results}

In the 21 studies selected for review (see Table 1), there are a number of studies whose samples overlap with one another. There are six reporting on various stages of a longitudinal study involving two sites. Three of the studies (Guilfoyle, Crimmins, \& Hood, 2011; McGrady, Peugh, \& Hood, 2014; Wu, Hilliard, Rausch, Dolan, \& Hood, 2013) report data from one location and the others report results from both locations (McGrady, Laffel, Drotar, Repaske, \& Hood, 2009; Herzer, Vesco, Ingerski, Dolan, \& Hood, 2010; Sander, Odell, \& Hood, 2010). For the purposes of this review, these will be considered as one study, referred to as the Depression \& Diabetes study. The two studies by Austin et al. (Austin, Guay, Senecal, Fernet, \& Nouwen, 2013; Austin, Senecal, Guay, \& Nouwen, 2011) will be referred to as the Dietary Self-Efficacy Study and the two studies by Skinner and Hampson (1998, 2001) will be referred to as the Personal Models study. This left 14 distinct studies for review.

Results of the validity assessment indicated that overall study validity was variable. Recruitment methods were similar across studies and primarily involved convenience samples from outpatient clinics. However, there was more variability in data collection methods and sample sizes. Bespoke, study-specific questionnaires were used in a number of studies - in some cases, measures were completely new, while in others, researchers created their own adaptations of existing measures. Consequently, validity information for many of these measures was either not available or not provided. Sample sizes ranged from 28 to 289 with sample size calculations provided in only a few cases. Information regarding measures 
used, sample size and limitations is provided in Table 1 to inform analysis. Validity information will be used in the discussion to help with interpretation, particularly in cases of equivocal findings.

-Table 1 here-

Across the 14 studies, nine different measures were used for self-management. The most frequently investigated self-care outcome was frequency of blood glucose monitoring (BGM). Where this was not obtained by meter download, medical charts or other form of self-report, adolescents provided this information via self-care adherence measures. These measures typically ask adolescents how adherent they have been to BGM as well as insulin, diet and exercise recommendations over a recent period of time. In terms of psychological factors, only four studies used the same measures as another.

Table 2 includes study findings which will also be referred to below. All relevant correlations were extracted from the included papers; moderate to strong correlations $(\mathrm{r}=.3$ and above: Cohen, 1992) are included in discussion regardless of the statistical significance in their study of origin (Sullivan \& Feinn, 2012). Data from Time 1 is presented for longitudinal studies. The results are organised by psychological variable, and under the broader categories of emotional and cognitive.

-Table 2 here-

\section{Emotional Variables}

Depression. Data from the Depression \& Diabetes study found a small, negative relationship between depressive symptoms and frequency of BGM (average correlation =.21) (Herzer et al., 2011; Wu et al., 2013), and in regression analyses, adolescents reporting more depressive symptoms reported less frequent BGM (McGrady et al., 2009; Guilfoyle et al.; 2011). 
Anxiety. Anxiety, in various forms, was addressed by three studies (DiBattista, Hart, Greco \& Glozier, 2009; Herzer et al., 2011; Skinner \& Hampson, 2011). State and trait anxiety were found to negatively correlate with frequency of BGM, though correlations are small (-.25 and -.17) (Herzer et al., 2011). Anxiety has also been found to relate to dietary adherence ( $r=.23$; Skinner \& Hampson, 2001).

DiBattista et al. (2009) reported analysis separately for females and males due to hypothesised differences in levels of social anxiety. Social anxiety was related to insulin $(\mathrm{r}=$ -.39) and dietary adherence for males $(r=-.50)$ but correlations with BGM and exercise adherence were negligible for males, and small for females. The largest effect for females was found between social anxiety and insulin adherence $(r=.21)$.

Fear of hypoglycaemia. DiBattista et al. (2009) also examined the relationships between fear of hypoglycaemia and self-management behaviours. For females, fear of hypoglycaemia was negligibly related to insulin adherence, glucose testing and exercise adherence, but showed a small, negative correlation with diet. There was a moderate relationship between adherence to insulin regime and fear of hypoglycaemia in males $(\mathrm{r}=-$ .38). Additionally, increased fear of hypoglycaemia also related to diet $(r=-.27)$ and exercise $(r=-.16)$ though these correlations were small.

Stress. Two studies looked at different aspects of stress - one included life stress and diabetes stress (Farrell, Hains, Davies, Smith, \& Parton, 2004) while the other focused on diabetes stress alone (Bennett Murphy, Thompson, \& Morris, 1997). In a path analysis, life stressors and frequency of BGM had a statistically significant path coefficient. There was no direct relationship found between diabetes-specific stress and frequency of BGM. This contrasts with the findings of Bennett Murphy et al. (1997) who found a moderate relationship between diabetes-specific stress and frequency of BGM $(r=-.38)$. 
Diabetes distress. Diabetes distress refers to 'the unique, often hidden emotional burdens and worries that are part of the spectrum of patient experience when managing a severe, demanding chronic disease like diabetes’ (Fisher, Hessler, Polonsky, \& Mullan, 2012, p. 259) and was studied by Nouwen et al. (2009) in the context of diet adherence correlations were negative but small.

Summary of emotional variables. While many relationships have been demonstrated between emotional variables and self-management behaviour, the strongest effect was found for social anxiety and adherence to diet in males. Diabetes stress is moderately related to frequency of BGM, while fear of hypoglycaemia is moderately related to insulin adherence for males.

\section{Cognitive Variables}

Self-efficacy. The research identified in this review addressed self-efficacy in regards to diet and overall management of diabetes.

Perceived dietary self-efficacy. Two studies examined self-efficacy in relation to adherence to dietary advice. Nouwen et al. (2009) found that dietary self-efficacy was positively and moderately related to diet adherence. This was corroborated by The Dietary Self-Efficacy Study which also reported that self-efficacy was positively related to diet adherence with correlations ranging from .31 to .56.

Diabetes self-efficacy. Overlapping dietary self-efficacy is the more general concept of diabetes self-efficacy. The Diabetes \& Depression study found that diabetes self-efficacy was positively correlated with frequency of BGM $(r=0.21)$.

Motivation. The Dietary Self-Efficacy Study also examined the adolescents’ motivation in regards to their dietary adherence and found moderate to strong correlations (average $r=.46$ ). 
Running head: Psychological factors and adolescent diabetes

Support for autonomy. Adolescents’ perceived support from parents and health care providers for autonomy in regards to dietary self-care was also investigated by the Dietary Self-Efficacy study. Correlations for perceived autonomy support from parents and dietary self-care ranged from .27 to .51 (average $r=.39$ ) whereas correlations for perceived autonomy support from health care providers were .19 to .40 (average $r=.30$ ).

Social context for BGM. Borus et al. (2013) conducted a unique study examining the influence of social context and related cognitions and emotions on BGM behaviour. Participants were more likely to BGM when they had a strong desire to blend in (OR $=9.13$, approximate $r=.68$ ). Participants were less likely to BGM when they had a strong desire to impress those around them $(\mathrm{OR}=.23$, approximate $\mathrm{r}=-.50)$.

Diabetes illness representations. Illness representations (alternately referred to as perceptions, beliefs and personal models) are cognitions an individual has regarding different aspects of an illness. According to the Common Sense Model (Leventhal, Nerenz, \& Steele, 1984), these cognitions influence coping behaviours such as self-management behaviours. Illness representations were addressed by 5 studies using 7 different measures (in whole or part). Only two studies used items from the same measure (Personal Models study; Nouwen et al., 2009); however, several of the subscales of the different measures are conceptually similar.

One study found that perceived threat to health from diabetes was negatively correlated with BGM from meter download $(r=-.27)$ as well as the blood glucose subscale on the SCI ( $r=$-.39) (McGrady et al, 2014). Perceived threat was also negatively related to the SCI insulin/food subscale $(r=-.22)$ which addresses insulin and food regulation. Regarding perceived treatment effectiveness to control diabetes, one study (McGrady et al., 2014) found small, positive correlations with blood glucose adherence (as measured by SCI), another study found correlations with actual frequency of BGM $(r=.23)$ 
(Personal Models study), and two found moderate correlations with dietary self-care $(r=.31$, Nouwen et al., 2009; $\mathrm{r}=.46$, Personal Models study). For treatment effectiveness to prevent complications, one study found correlations with the BGM subscale (McGrady et al., 2014), and another (Skinner \& Hampson, 1998) with actual BGM frequency $(r=.25)$ and dietary self-care $(r=.37)$. For the perceived impact of diabetes on one's life, one study found that when the impact is reported to be more significant, dietary self-care is poorer (Nouwen et al., 2009; $r=-.20)$. Using a longitudinal design, Skinner and Hampson (2001) found that change in perceived effectiveness of treatment to control diabetes was moderately related to diet adherence.

Serrabulho et al. (2012) reported a small relationship between more positive representations about diabetes and better adherence to physical exercise $(r=.16)$. Patino and colleagues (2005) examined whether health beliefs (about perceptions of susceptibility to complications, severity of diabetes, benefits of adherence, costs of non-adherence and cues for adherence action) would predict adherence in ethnic minority adolescents. No significant associations were found between health beliefs and adherence (correlations were not reported).

In summary, illness representations appear to be important for self-care behaviour with correlations ranging from small to moderate. Perceptions of higher threat are moderately linked to less frequent BGM and less belief in treatment effectiveness is moderately linked to poorer dietary self-care.

Attributional style. Bennett Murphy et al. (1997) found a negative, moderate correlation $(r=-.36)$ between attributional style for negative events and frequency of BGM - the more external, stable and global the attributions for negative events, the less frequently BGM occurred. The correlation between attributional style for positive events and frequency of BGM was small $(r=.16)$. 
Decision-making. Miller and Drotar (2007) looked at aspects of adolescents’ decision-making process using the Melbourne Decision-Making Questionnaire (MDMQ). The MDMQ results in 4 subscale scores - one (vigilance) which reflects competent decision making and three which reflect different styles of maladaptive decision-making (hypervigilance, buck-passing and procrastination). Small correlations were found between frequency of BGM and hypervigilance. Decision-making was examined as a potential mediator between parent-adolescent communication and frequency of BGM but it did not meet the necessary conditions.

Locus of control. Locus of control was investigated by Bennett Murphy and colleagues (1997) as findings in prior research were contradictory. Internal control when well correlated moderately with frequency of BGM whereas control when ill showed a small relationship to BGM. In a hierarchical regression model, perceived control when ill explained $10 \%$ of the variance (entered after demographic, family functioning, and self-esteem variables).

Compensatory beliefs. Rabiau and colleagues (2009) explored beliefs about how engaging in one activity can counteract the negative effects of another - specifically, how not engaging in BGM can be justified under certain conditions (e.g. 'I do not have to test my glucose regularly if my meals are carefully planned by my parents'). They found that the more participants endorsed glucose testing compensatory beliefs (CBs), the lower their adherence to glucose testing $(r=-.49)$. Furthermore, CBs explained an additional $10 \%$ of the variance in adherence to BGM in a regression model which already included demographic variables, diabetes knowledge, treatment effectiveness beliefs, and perceived competence.

Perceived social support. Skinner and Hampson (1998) observed that general family support was moderately correlated with diet in the positive direction. The relationships between general family support and BGM testing and adherence to insulin therapy were 
small. Small relationships were also demonstrated for general friend support with diet and insulin adherence.

Adjustment. One study reported that psychological adaptation to diabetes was positively but weakly related to exercise adherence (Serrabulho et al., 2012). Because a purpose-designed questionnaire was used, it was not possible to determine how psychological adaptation was operationalised.

Self-esteem. Bennett Murphy et al. (1997) examined self-esteem and found moderate correlations for both global self-esteem $(r=.39)$ and self-esteem related to appearance with frequency of BGM $(r=.42)$.

Summary of cognitive variables. Findings indicate that more frequent BGM is moderately associated with lower perceived threat, an internal attributional style for negative events, greater perception of internal control over diabetes when well, fewer compensatory beliefs and higher self-esteem.

Better dietary adherence is strongly related to greater intrinsic motivation. Moderate relationships were found between better dietary adherence and greater dietary self-efficacy, greater perceived support for autonomy for diet choices, greater belief in treatment effectiveness to control diabetes and prevent complications and increased general family support.

In terms of social situations, adolescents are more likely to complete BGM if they desire to blend in, and less likely to complete it when they want to impress their companions. Other Variables

Quality of life (QoL). One study examined diabetes-related QoL (DiBattista et al., 2009). Correlations between QoL and adherence to insulin regime, BGM and diet were small for females. For males, QoL was moderately related to adherence to insulin regime $(r=-.44)$ and diet $(r=-.27)$. 
Personality. Two studies (Waller et al., 2013; Wheeler, Wagaman, \& McCord, 2012) investigated the relationships between self-management and the five personality factors of conscientiousness, openness to experience, extraversion, agreeableness and neuroticism/emotional regulation. Strong correlations were observed for four of the personality factors (Wheeler et al., 2012): higher levels of conscientiousness with greater adherence to insulin administration and dietary advice; higher levels of agreeableness with greater adherence to insulin administration; greater neuroticism with lower levels of insulin adherence; and higher levels of extraversion with greater adherence to exercise. Moderate relationships were reported for extraversion and BGM frequency; agreeableness $(r=.43)$ and neuroticism $(r=-.31)$ to diet adherence and neuroticism $(r=-.31)$ and openness to experience $(r=.30)$ for exercise. In a hierarchical regression model including age, conscientiousness and previous BGM frequency, conscientiousness independently predicted BGM, explaining 5\% of the variance (Waller et al., 2013).

Variables with evidence demonstrating a moderate or strong effect size are summarised in Table 3.

\section{Discussion}

This review demonstrates the importance of psychological variables in selfmanagement for adolescents with Type 1 diabetes. Relationships have been demonstrated for emotional, cognitive and personality variables with frequency of BGM, diet, exercise and insulin adherence (see Table 3).

Emotional variables demonstrated some conflicting findings, particularly for anxiety and stress. Anxiety was found to positively correlate with dietary adherence in one study (Personal Models study); however, when considering the limitations of this research, it is possibly more likely that anxiety is associated with poorer self-management. The differences in findings regarding diabetes and life stress could be explained by use of a purpose-designed 
measure and smaller sample size for one study (Bennett Murphy et al., 1997). It is also possible that the measure for diabetes stress used in this study (Bennett Murphy et al., 1997) overlapped with life stress, which was shown to have a direct relationship to frequency of BGM (Farrell et al., 2004). Therefore, it may be more likely that diabetes specific stress does not have a direct effect on frequency of BGM. The strongest negative effects for emotional variables on self-management were found for increased social anxiety and fear of hypoglycaemia. This suggests that interventions designed to improve self-management should consider these variables in particular. However, there is a paucity of research on the relationship between emotional variables and self-management in diabetes. This might be because emotional variables are more often treated as the outcomes in psychosocial research in diabetes.

There is a greater quantity of evidence for cognitive variables, especially for illness representations which were the most frequently studied psychological variable. However, most variables were only examined in one study. The strongest effect sizes on selfmanagement were found in the Dietary Self-Efficacy study and one study examining the effect of compensatory beliefs. Findings from the Dietary Self-Efficacy study suggest that support for adolescents’ autonomy and intrinsic motivation are important for dietary self-care behaviour. A recent study has also highlighted that peer support that might be perceived as threatening autonomy leads to poorer glycaemic control (Doe, 2016). Additionally, compensatory beliefs may also be important for consideration in intervention design.

Strong correlations were also observed between greater conscientiousness and better diet adherence, as well as greater neuroticism and poorer insulin adherence. While these were found in a study with notable limitations (Table 1), the association between conscientiousness and health is well-established (Bogg \& Roberts, 2004). However, whether conscientiousness is an appropriate target for intervention, over and above interventions seeking to improve 
health behaviours, is debatable (English \& Carstensen, 2014). Indeed, personality traits in general are difficult to modify and interventions designed to modify personality traits linked to health problems have used health behaviours as their focus (Magidson et al., 2014).

The ability of this review to draw firm conclusions has been hampered by the heterogeneity of the research. While there is evidence for relationships, there is not enough corroboration by similar studies to provide confidence in the results. There are a number of ways future research could strengthen the evidence base. Firstly, there are two identified evidence gaps. There was no evidence in this review for the relationship between exercise behaviour and emotional variables. Emotional variables such as depression (Vickers, Nies, Patten, Ross, \& Smith, 2006) and anxiety (Lawton, Ahmad, Hanna, Douglas \& Hallowell, 2005) have been shown to be important for exercise behaviour in type 2 diabetes, suggesting there may also be relevance for individuals with type 1 diabetes. Evidence was found for the importance of intrinsic motivation and of support for autonomy for diet adherence. Whether or not this applies to other self-care behaviours has not been investigated and may be a fruitful area for further research.

Secondly, a number of studies used global adherence measures which excluded them from this review. Global adherence measures obscure the underlying relationships which, arguably, provide the specific information necessary for theory development and intervention design. Adherence is not a unidimensional construct (Dunbar-Jacob \& Mortimer-Stephens, 2001); therefore, future research should report subscale information and measure behaviours individually. Research should also include BGM frequency, preferably by download from meters. More frequent BGM is associated with lower $\mathrm{HbA}_{1 \mathrm{C}}$ (Karter et al., 2001) and selfmonitoring of blood glucose provides vital feedback to facilitate decisions regarding insulin administration which is crucial for good blood sugar control (Miller et al., 2013). 
Running head: Psychological factors and adolescent diabetes

Lastly, there is a need for longitudinal studies, replication and use of standardised measures. More longitudinal research would help elucidate the direction of the effects noted. Studies should also utilise similar, standardised measures, for both psychological variables and self-care behaviours.

In addition, this review has purposely not examined the relationship between selfmanagement behaviour in adolescents and the psychosocial variables that 'reside' within the parent (eg. Pate et al., 2016) or the qualitative experiences of adolescents (eg. Ferrari et al., 2016). These are areas worthy of further investigation in terms of informing psychosocial interventions.

\section{Conclusions}

This review has found some evidence that a number of psychological factors are associated with self-care behaviours; however, this is often on the basis of individual studies rather than a number of studies using the same measures obtaining similar findings. Studies not only addressed different topics, but even where the same topics were examined, different, often new, measures were used, negatively affecting the validity of the evidence. In order to facilitate further development of theory for adolescents with type 1 diabetes, future studies should attempt replication and employ similar, standardised measures where possible. 
Running head: Psychological factors and adolescent diabetes

\section{References}

Austin S, Guay F, Senecal C, Fernet C and Nouwen A (2013) Longitudinal testing of a dietary self-care motivational model in adolescents with diabetes. Journal of Psychosomatic Research 75: 153-159.

Austin S, Senecal C, Guay F and Nouwen A (2011) Effects of gender, age, and diabetes duration on dietary self-care in adolescents with type 1 diabetes: A self-determination theory perspective. Journal of Health Psychology 16: 917-928.

Ayling K, Brierley S, Johnson B, Heller S and Eiser C (2014) Efficacy of theory-based interventions for young people with Type 1 diabetes. British Journal of Health Psychology 20: 428-446.

Bandura A (1982) The assessment and predictive generality of self-percepts of efficacy. Journal of Behavioural Therapy \& Experimental Psychiatry 13: 195-199.

Bennett Murphy LM, Thompson Jr RJ and Morris MA (1997) Adherence behaviour among adolescents with Type 1 insulin-dependent diabetes mellitus: The role of cognitive appraisal processes. Journal of Pediatric Psychology 22: 811-825.

Bogg T and Roberts BW (2004) Conscientiousness and health-related behaviours: A metaanalysis of the leading behavioural contributors to mortality. Psychological Bulletin 130: 887-919.

Borus JS, Blood E, Volkening LK, Laffel L and Shrier LA (2013) Momentary assessment of social context and glucose monitoring adherence in adolescents with type 1 diabetes. Journal of Adolescent Health 52: 578-583.

Cohen J (1992) A power primer. Psychological Bulletin 112: 155-159.

DiBattista AM, Hart TA, Greco L and Glozier J (2009) Type 1 diabetes among adolescents: Reduced diabetes self-care caused by social fear and fear of hypoglycaemia. The Diabetes Educator 35: 465-475. doi: 10.1177/0145721709333492 
Running head: Psychological factors and adolescent diabetes

Doe E (2016) An analysis of the relationships between peer support and diabetes outcomes in adolescents with type 1 diabetes. Journal of Health Psychology. doi: $10.1177 / 1359105316656228$

Dunbar-Jacob J and Mortimer-Stephens MK (2001) Treatment adherence in chronic disease. Journal of Clinical Epidemiology 54: S57-S60.

Effective Public Health Practice Project (2009) Quality Assessment Tool for Quantitative Studies. Ontario: EPHPP. Available from: http://www.ephpp.ca/tools.html

English T and Carstensen L (2014) Will interventions targeting conscientiousness improve aging outcomes? Developmental Psychology 50: 1478-1481. doi: 10.1037/a0036073

Farrell SP, Hains AA, Davies WH, Smith P and Parton E (2004) The impact of cognitive distortions, stress, and adherence on metabolic control in youths with Type 1 diabetes. Journal of Adolescent Health 34: 461-467.

Ferrari M, McIlwain DJF, Ambler G (2016) A qualitative comparison of needles and insulin pump use in children with type 1 diabetes. Journal of Health Psychology. doi: $10.1177 / 1359105316653999$

Fisher L, Hessler DM, Polonsky WH and Mullan J (2012) When is diabetes distress clinically meaningful? Establishing cut points for the Diabetes Distress Scale. Diabetes Care 35: 259-264.

Guilfoyle SM, Crimmins NA and Hood KK (2011) Blood glucose monitoring and glycemic control in adolescents with type 1 diabetes: Meter downloads versus self-report. Pediatric Diabetes 12: 560-566.

Hampson SE, Skinner TC, Hart J, Storey L, Gage H, Foxcroft D, Kimber A, Shaw K and Walker J (2001) Effects of educational and psychosocial interventions for adolescents with diabetes mellitus: a systematic review. Health Technology Assessment 5: 10. 
Running head: Psychological factors and adolescent diabetes

Health \& Social Care Information Centre (2013) National Diabetes Audit 2012-2013 Report

1: Care Processes and Treatment Targets. Retrieved from

http://www.hscic.gov.uk/catalogue/PUB14970/nati-diab-audi-12-13-care-proc-rep.pdf

Herzer M, Vesco A, Ingerski LM, Dolan LM and Hood KK (2011) Explaining the family conflict-glycemic control link through psychological variables in adolescents with type 1 diabetes. Journal of Behavioural Medicine 34: 268-274.

Hilliard ME, Wu YP, Rausch J, Dolan LM and Hood KK (2013) Predictors of deteriorations in diabetes management and control in adolescents with Type 1 Diabetes. Journal of Adolescent Health 52: 28-34.

Karter AJ, Ackerson LM, Darbinian JA, D’Agostino RB, Ferrara A, Liu J and Selby JV (2001) Self-monitoring of blood glucose levels and glycemic control: The Northern California Kaiser Permanente diabetes registry. The American Journal of Medicine 111: 2-9.

Lawton J, Ahmad N, Hanna L, Douglas M and Hallowell N (2005). 'I can’t do any serious exercise’: Barriers to physical activity amongst people of Pakistani and Indian origin with Type 2 diabetes. Heath Education Research 21: 43-54.

Leventhal H, Nerenz DR and Steele DJ (1984) Illness representation and coping with health threats. In: Baum A, Taylor SE and Singer JE (eds), Handbook of Psychology and Health. Hillsdale, NJ: Lawrence Erlbaum Associates, pp. 219- 252.

Lind M, Odén A, Fahlén M and Eliasson B (2009) The True Value of HbA1c as a Predictor of Diabetic Complications: Simulations of HbA1c Variables. PLoS ONE 4: e4412.

Magidson JF, Roberts BW, Collado-Rodriguez A and Lejuez CW (2014) Theory-driven intervention for changing personality: expectancy value theory, behavioral activation and conscientiousness. Developmental Psychology 50: 1442-1450. 
Running head: Psychological factors and adolescent diabetes

McGrady ME, Laffel L, Drotar D, Repaske D and Hood KK (2009) Depressive symptoms and glycemic control in adolescents with Type 1 diabetes. Diabetes Care 32: 804-806. McGrady ME, Peugh JL and Hood KK (2014) Illness representations predict adherence in adolescents and young adults with type 1 diabetes. Psychology \& Health 29: 985-998.

Miller KM, Beck RW, Bergenstal RM et al. (2013) Evidence of a strong association between frequency of self-monitoring of blood glucose and hemoglobin $\mathrm{A}_{1 \mathrm{c}}$ levels in T1D exchange clinic registry participants. Diabetes Care 36: 2009-2014.

Miller VA and Drotar D (2007) Decision-making competence and adherence to treatment in adolescents with diabetes. Journal of Pediatric Psychology 32: 178-188. doi: 10.1093/jpepsy/jsj122

Murphy HR, Rayman G and Skinner TC (2006) Psycho-educational interventions for children and young people with type 1 diabetes. Diabetic Medicine 23: 935-943.

Neylon OM, O'Connell MA, Skinner TC and Cameron FJ (2013) Demographic and personal factors associated with metabolic control and self-care in youth with type 1 diabetes: A systematic review. Diabetes/Metabolism Research Reviews 29: 257-272.

Nouwen A, Urquhart Law G, Hussain S, McGovern S and Napier H (2009) Comparison of the role of self-efficacy and illness representations in relation to dietary self-care and diabetes distress in adolescents with Type 1 diabetes. Psychology \& Health 24: 10711084.

Pate T, Klemencic S, Battelino T, Bratina N (2016) Fear of hypoglycemia, anxiety, and subjective well-being in parents of children and adolescents with type 1 diabetes. Journal of Health Psychology. doi: 10.1177/1359105316650931

Patino AM, Sanchez J, Eidson M and Delamater AM (2005) Health beliefs and regimen adherence in minority adolescents with Type 1 diabetes. Journal of Pediatric Psychology 30: 503-512. doi:10.1093/jpepsy/jsi075 
Running head: Psychological factors and adolescent diabetes

Patterson CC, Gyürüs E, Rosenbauer J et al. (2012) Trends in childhood type 1 diabetes incidence in Europe during 1989-2008: evidence of non-uniformity over time in rates of increase. Diabetologia 55: 2142-2147

Peyrot M and Rubin RR (2007) Behavioural and psychosocial interventions in diabetes: A conceptual review. Diabetes Care 30(10): 2433-2440. doi: 10.2337/dc07-1222

Rabiau MA, Knauper B, Nguyen T-K, Sufrategui M and Polychronakos C (2009)

Compensatory beliefs about glucose testing are associated with low adherence to treatment and poor metabolic control in adolescents with Type 1 diabetes. Health Education Research 24: 890-896.

Sander EP, Odell S and Hood KK (2010) Diabetes-specific family conflict and blood glucose monitoring in adolescents with Type 1 diabetes: Mediational role of diabetes selfefficacy. Diabetes Spectrum 23: 89-94.

Serrabulho M, Matos MG and Raposo J (2012) The health and lifestyles of adolescents with type 1 diabetes in Portugal. European Diabetes Nursing 9: 12-16.

Skinner TC and Hampson SE (1998) Social support and personal models of diabetes in relation to self-care and well-being in adolescents with Type 1 diabetes mellitus. Journal of Adolescence 21: 703-715.

Skinner TC and Hampson SE (2001) Personal models of diabetes in relation to self-care, well-being, and glycemic control: A prospective study in adolescence. Diabetes Care 24: 828-833.

Sullivan GM and Feinn R (2012) Using effect size — or why the P value is not enough. Journal of Graduate Medical Education 4: 279-282. 
Running head: Psychological factors and adolescent diabetes

Vickers KS, Nies MA, Patten CA, Ross D and Smith SA (2006) Patients with diabetes and depression may need additional support for exercise. American Journal of Health Behaviour 30: 353-362.

Waller D, Johnston C, Molyneaux L, Brown-Singh L, Hatherly K, Smith L, et al. (2013). Glycemic control and blood glucose monitoring over time in a sample of young australians with type 1 diabetes: The role of personality. Diabetes Care 36: 29682973.

Wheeler K, Wagaman A and McCord D (2012) Personality traits as predictors of adherence in adolescents with type I diabetes. Journal of Child \& Adolescent Psychiatric Nursing 25: 66-74.

Wu YP, Hilliard ME, Rausch J, Dolan LM and Hood KK (2013) Family involvement with the diabetes regimen in young people: The role of adolescent depressive symptoms. Diabetic Medicine 30: 596-602. 